\title{
O uso do GeoGebra mobile como ferramenta auxiliar no ensino de funções inversas e logarítmicas
}

\section{The use of GeoGebra mobile as auxiliary tool in teaching of inverse and logarithmic functions}

\begin{abstract}
Murilo Carvalho Feitosa Universidade Federal Rural do Semi-árido (UFERSA), Iniciação Científica do Conselho Nacional de Desenvolvimento Científico e Tecnológico (CNPq), Pau dos Ferros, RN, Brasil http://orcid.org/0000-0003-1163-8838, murilocfeitosa@gmail.com
\end{abstract}

\section{Adelmo Artur de Aquino \\ Universidade do Estado do Rio Grande do Norte (UERN), Programa de Pós-graduação em Ensino (PPGE), Pau dos Ferros, RN, Brasil http://orcid.org/0000-0002-2119-1825, artur-aquino1@hotmail.com}

\author{
Bruno Fontes de Sousa \\ Universidade Federal Rural do Semi-árido (UFERSA), Departamento de Ciências Exatas e \\ Naturais (DCEN), Pau dos Ferros, RN, Brasil \\ http://orcid.org/0000-0003-1359-0065, bruno.fontes@ufersa.edu.br
}

\begin{abstract}
Otávio Paulino Lavor Universidade Federal Rural do Semi-árido (UFERSA), Departamento de Ciências Exatas e Naturais (DCEN), Pau dos Ferros, RN, Brasil http://orcid.org/0000-0001-5237-3392, otavio.lavor@ufersa.edu.br
\end{abstract}

\section{Informações do Artigo \\ Como citar este artigo \\ FEITOSA, Murilo Carvalho; AQUINO, Adelmo Artur de; SOUSA, Bruno Fontes de; LAVOR, \\ Otávio Paulino. O uso do GeoGebra como ferramenta auxiliar no ensino de funções inversas e logarítmicas. REMAT: Revista Eletrônica da Matemática, Bento \\ Gonçalves, RS, v. 6, n. 2, p. e2003, 18 ago. 2020. DOI: \\ https://doi.org/10.35819/remat2020v6i2id3952}

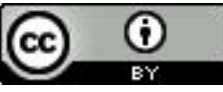

Histórico do Artigo

Submissão: 14 de março de 2020

Aceite: 4 de junho de 2020.

\section{Resumo}

O processo de ensino e aprendizagem de alguns conceitos enfrenta algumas dificuldades que podem estar associadas à motivação ou à interação. Como exemplo, tem-se as funções inversas e logarítmicas que possuem papel fundamental para a apropriação de conhecimentos do Cálculo Diferencial e Integral. Nesse sentido, essa pesquisa investiga a apresentação desses conteúdos por meio do GeoGebra a alunos ingressantes em um curso de Ciência e Tecnologia. Na metodologia adotada, o conteúdo é apresentado paralelamente ao uso do GeoGebra para a visualização do comportamento das funções abordadas e cada discente, em seu smartphone, acompanha o que é ministrado utilizando o aplicativo. Durante a intervenção, foi possível perceber a interação e a motivação do público envolvido e as avaliações apontam para um bom desempenho, mostrando que as tecnologias podem ser aliadas ao processo de ensino e aprendizagem.

\section{Palavras-chave}

GeoGebra Mobile

Ensino e Aprendizagem

Motivação

\section{Keywords}

GeoGebra Mobile

Teaching and Learning

Motivation

\begin{abstract}
The process of teaching and learning of some concepts faces some difficulties that may be associated with motivation or interaction. As an example, there are the inverse and logarithmic functions that have a fundamental role in the appropriation of knowledge of differential calculus. In this sense, this work investigates the presentation of these contents through GeoGebra
\end{abstract}


to a Science and Technology course entering students. The content is submitted in parallel with the use of GeoGebra to visualize the behavior of the addresed functions and each student, on their smartphone, follows everything that is taught using the application. During the intervention, it was possible to notice the interaction and motivation of the public involved and the evaluations point to a good performance, showing that technologies can be allies in teaching and learning process.

\section{Introdução}

A Matemática, aos olhos de quem vê, está presente em todos os fenômenos da natureza, sendo esses traduzidos por meio de seus padrões, simetrias e peculiaridades, que em muitos casos são palpáveis à sensibilidade humana. Dizer que basta olharmos ao nosso redor para notarmos sua presença em todas as coisas, como na geometria e comprimento dos objetos, massa, tempo, por exemplo, caracteriza-se como um discurso superficial se levarmos em consideração a deficiência na formação matemática dos alunos na atual conjectura educacional do Brasil.

Embora a formação científica seja imprescindível para o cidadão moderno, é inato ao ser humano a necessidade de relacionar os acontecimentos naturais ao seu cotidiano, essencialmente à sua vivência em coletividade, sendo que tal necessidade é observada desde os primórdios. A busca pela tradução e representação dessas situações, muitas vezes observada em padrões, instigou o interesse do homem pelos números, levando, consequentemente, à criação de padrões e sistemas numéricos, e pelas formas de utilizá-los para representar diferentes situações.

A Matemática pode ser considerada como a ciência mais relevante da sociedade moderna, sendo estudada desde os anos iniciais do ensino, preparando as crianças em diferentes aspectos, sejam cognitivos, motores, ou àqueles voltados à formação para o mercado de trabalho. Em termos mais avançados, a Matemática está intrinsicamente ligada à pesquisa científica, à investigação de situações e fatos de origem natural ou artificiais, com o intuito de descobrir o novo, tudo isso a partir de uma ciência fundamentada em uma linguagem criteriosamente lógica e consistente.

No que tange às práticas didático-pedagógicas empregadas no ensino de ciências exatas frente à sociedade tecnológica, elas levantam fortes questionamentos pedagógicos, não apenas no Ensino Superior, mas em todos os níveis de ensino (TEDESCO, 2004 apud FRANCO; PORTO; ALMEIDA, 2016, p. 14). Reflexões acerca das lacunas observadas são comuns no âmbito educacional, em nível de ensino e de aprendizagem das disciplinas e conteúdos relacionados a essa área do conhecimento. A ausência de metodologias eficazes e acessíveis que façam uso de recursos computacionais é outra variável recorrente. Diante disso, nota-se um cenário preocupante no qual se evidencia a escassez no emprego dos mecanismos de forma a encabeçar reflexões e estratégias de planejamento direcionadas à aprendizagem significativa dos conteúdos. 
Nesse sentido, evidencia-se a emergente necessidade de adaptação das instituições de ensino quanto à utilização das Tecnologias da Informação e Comunicação (TIC's). Como afirma Nascimento (2012), as tecnologias vem causando uma reestruturação do método tradicional de ensino. A não adesão às ferramentas digitais acarretam em um fracasso natural das metas de ensino-aprendizagem enfraquecendo, consequentemente, ainda mais os métodos tradicionais de ensino que por si só não serão capazes de alcançar as metas desejadas (FAVA, 2012). As TIC's estão cada vez mais presentes no nosso cotidiano, razão pela qual ocupam função imprescindível para a educação. Pesquisas acerca das TIC's e suas formas de utilização em sala de aula destacam a sua relevância no ensino de Matemática, sendo também um forte indicador de competência a ser trabalhada na formação inicial dos professores (LOPES, 2011, p. 2).

À vista dessas circunstâncias descritas, e fundamentando-se nos discursos dos teóricos estudados, bem como em pesquisas atuais que abordam o uso das TIC's, acreditamos ser possível contornar esses cenários questionando as abordagens e possibilidades metodológicas do ensino de Matemática, em particular do Cálculo Diferencial e Integral e matérias afins, quando indagamos: Por que os estudantes do Ensino Superior veem o estudo da Matemática como uma tarefa desinteressante, tediosa e de difícil aprendizagem? Em quais perspectivas é possível abordar os conceitos do Cálculo Diferencial e Integral, especificamente das funções inversas e logarítmicas, frente às novas tecnologias? Como afirma Mattar (2013), há uma necessidade de novas estratégias pedagógicas para dar conta da interação, comunicação e produção de conteúdo colaborativo em ambientes virtuais, como por exemplo o Geogebra.

Mediante isso, a presente pesquisa tem o intuito de explorar a utilização das novas tecnologias, em especial o software matemático GeoGebra, que de acordo com Molinari, Santos e Retslaff (2019), facilita a interpretação gráfica de funções. E como afirma Araújo e Nóbriga (2010), uma das diferenças para outros softwares de Geometria Dinâmica é o fato de se poder acessar as funções, tanto via botões na Barra de Ferramentas, quanto pelo Campo de Entrada. Em sua versão mobile, gratuita e bastante intuitiva, abrange conteúdos desde disciplinas iniciais até disciplinas que exigem maior formalismo matemático nos cursos de ciências exatas. $O$ estudo tem como locus uma turma de Cálculo Diferencial e Integral I, mas vale ressaltar que o aplicativo que utilizamos abrange outras disciplinas do Ensino Superior, como Geometria Analítica e Cálculo Diferencial e Integral II. Em linhas gerais, a pesquisa objetiva analisar se o aplicativo GeoGebra mobile cumpre o requisito de ser uma ferramenta eficiente no auxílio da aprendizagem do aluno, mais precisamente, aplicado a funções inversas e logarítmicas. De acordo com Petla (2008), o aluno é o mais interessado na utilização de tecnologias nesse processo de ensino-aprendizagem.

Alguns autores como Lopes e Santos (2016), Meier e Gravira (2012), reforçam que a ideia de utilizar o GeoGebra como recurso metodológico pode apresentar resultados satisfatórios nos processos de ensino e aprendizagem. Por exemplo, segundo Rezende, Pesco e Bortolossi (2012), o software GeoGebra apresenta uma excelente interface dinâmica entre os sistemas algébrico e 
geométrico de representações, também se apresentando como uma poderosa ferramenta para o estudo do comportamento variacional das funções reais. Os mesmos autores também aferem que a utilização de softwares tem sido muito expressiva em cursos e minicursos por professores de Matemática.

Um dos principais motivos dessa pesquisa também diz respeito ao alto índice de reprovação na disciplina de Cálculo Diferencial e Integral I, e se refere aos alunos dos cursos de graduação de uma determinada universidade localizada no Semiárido Potiguar. Outro fator propulsor para a realização desse levantamento se deu pela nossa convivência e imersão na realidade citada, bem como por sondagens feitas em sala para com os discentes. Além disso, consideramos que atualmente existem muitos recursos que podem proporcionar melhores condições de ensino-aprendizagem, assim como abordado por Feitosa, Aquino e Lavor (2020), que utilizam o mesmo aplicativo como auxiliar no ensino de retas e planos, inclusive na procura por novos métodos que ajudem a obter uma maior eficiência na aprendizagem do aluno, de forma que o professor possa desempenhar melhor o seu papel frente à caraterística de agente mediador ou facilitador da aprendizagem.

\section{Funções inversas e logarítmicas e o uso do Geogebra}

Trabalharemos aqui com as funções inversas e os logaritmos. Começamos apresentando a definição de função:

Dados conjuntos $X$ e $Y$, uma função $f: X \rightarrow Y$ (lê-se 'uma função de $X$ em $Y$ ') é uma regra (ou conjunto de instruções) que diz como associar a cada elemento $x \in X$ um elemento $y=f(x) \in Y$. O conjunto $X$ chama-se domínio e $Y$ é o contra-domínio da função $f$. Para cada $x \in X$, o elemento $f(x) \in Y$ chama-se a imagem de $x$ pela função $f$ ou o valor assumido pela função $f$ no ponto $x \in X$. Escreve-se $x \mapsto f(x)$ para indicar que $f$ transforma (ou leva) $x$ em $f(x)$." (LIMA et al., 2016, p. 39).

Ainda de acordo com Lima et al. (2016, p. 42):

A natureza da regra que ensina como obter $f(x)$ quando $\mathrm{x}$ é dado é inteiramente arbitrária, sendo sujeita a apenas duas condições:

a) Não deve haver exceções: a fim de que a função tenha o conjunto $X$ como domínio, a regra deve fornecer $f(x)$ seja qual for $x \in X$ dado.

b) Não pode haver ambiguidades: a cada $x \in X$, a regra deve fazer corresponder um único $f(x)$ em $Y .[\ldots]$

Alguns exemplos de funções são:

1. As funções polinomiais $p: R \rightarrow R$, definidas pela regra

$$
p(x)=a_{0}+a_{1} x+a_{2} x^{2}+\cdots+a_{n-1} x^{n-1}+a_{n} x^{n},
$$

em que os símbolos $a_{0}, a_{1}, a_{2}, \cdots, a_{n-1}$ e $a_{n}$ (coeficientes do polinômio) são números reais arbitrários.

\section{As funções potências}

$$
f(x)=x^{a},
$$

em que a é um número real arbitrário. 
3. As funções exponenciais de base $a$, que são as funções

$$
f(x)=a^{x},
$$

em que a é um número real positivo e diferente de 1.

Considere uma função exponencial de base $a$. O domínio de $f$ é o conjunto dos números reais e a imagem de $f$ é o intervalo $(0,+\infty)$; se $0<a<1$, então $f$ é decrescente e se $a>1$, então $f$ é crescente. A função exponencial de base $e$, onde $e$ é o número de Euler, é denominada função exponencial natural (LIMA et al., 2016, p. 178).

Para definir funções logarítmicas é necessário introduzir o conceito de função inversa. De acordo com Lima et al. (2016, p. 192): Diz-se que a função $g: Y \rightarrow X$ é a inversa da função $f: X \rightarrow Y$ quando se tem
$g(f(x))=x$ e $f(g(y))=y$ para quaisquer $x \in X$ e $y \in Y$. Evidentemente, $g$ é
inversa de $f$ se, e somente se, $f$ é inversa de $g$.

Quando $g$ é a inversa de $f$, tem-se $g(y)=x$ se, e somente se, $f(x)=y$.

Os autores nos apresentam importantes propriedades a respeito das funções invertíveis (funções que admitem inversa). (i) Uma função $f: X \rightarrow Y$ é invertível se, e somente se, for bijetora. (ii) A inversa de uma função crescente é crescente e a inversa de uma função decrescente é decrescente. (iii) $O$ gráfico $G$ de uma função invertível $f: X \rightarrow Y$ e o gráfico $G^{\prime}$ de sua inversa $f^{-1}: Y \rightarrow X$, onde $X$ e $Y$ são conjuntos de números reais, são simétricos em relação à reta diagonal dos quadrantes ímpares do plano cartesiano $R^{2}$.

Em relação às funções exponenciais de base $a$, Lima et al. (2016) mostram que elas são invertíveis. Se $f(x)=a^{x}$, a inversa de $f$ é denominada função logarítmica de base $a$. Usamos a notação

$$
g(x)=\log _{a}(x)
$$

para a função logarítmica de base a. A inversa da função exponencial natural é denominada função logaritmo natural. Dado $x \in R$, o logaritmo natural de $x$ é denotado por $\ln (x)$. Se $0<a<$ $1, g$ é decrescente; se $a>1, g$ é crescente. Em particular, a função logaritmo natural é crescente.

As funções inversas e os logaritmos podem ser estudados com o auxílio do GeoGebra mobile, aplicativo adotado como principal recurso nesta pesquisa para ajudar em uma melhor qualidade de ensino e maior eficiência na aprendizagem. O GeoGebra é definido, de modo geral, como sendo um aplicativo de Matemática gratuito para todos os níveis de ensino, que funde geometria, álgebra, tabelas, gráficos, estatística e cálculo em uma única aplicação. Esse software é classificado como uma das TIC's, sendo uma das formas que os estudantes utilizam quando possuem dificuldades em Matemática. Dessa forma, tivemos a iniciativa de fazer o uso das TIC's em sala de aula, por meio do GeoGebra, para que os alunos já tivessem a noção de como e o que utilizar em caso de dúvidas em Matemática, já que muitos não possuem a sabedoria da existência de tal ferramenta. 


\section{Metodologia}

Com o intuito de mostrar a eficiência de um aplicativo gratuito e simples de ser utilizado, podendo auxiliar nos estudos dos alunos que cursam disciplinas das áreas de Ciências Exatas, aplicamos uma prática para o ensino de funções inversas e logarítmicas com o uso do GeoGebra mobile a alunos da disciplina de Cálculo Diferencial e Integral I de uma instituição de Ensino Superior da região do Semiárido Potiguar, no Rio Grande do Norte. A pesquisa foi realizada com 15 estudantes de uma turma de 29 jovens do Curso de Ciência e Tecnologia. O convite para participação na pesquisa foi realizado para toda a turma e aproximadamente $52 \%$ se voluntariaram a participar, demonstrando muita motivação.

A pesquisa consistiu de um pré-teste, a aplicação de uma sequência didática utilizando o GeoGebra mobile no ensino de funções inversas e logarítmicas e um pós-teste para a verificação da eficiência desta metodologia.

O pré-teste foi aplicado com toda a turma. Neste momento foi feita a seleção dos voluntários a partir de uma questão em que os alunos marcavam a opção se queriam se voluntariar ou não. Caso mais da metade da turma dissesse que sim, haveria um sorteio para que apenas a metade participasse. A turma foi dividida em dois grupos: um para estudar o conteúdo da forma tradicional e outro com o auxílio do GeoGebra mobile.

As perguntas do pré-teste foram as seguintes: Você já teve contato no Ensino Fundamental ou no Ensino Médio com funções inversas e logarítmicas? Seus professores do ensino básico utilizavam tecnologias para um melhor ensino-aprendizagem? Você tem conhecimento e domínio de funções inversas e logarítmicas? Você se disponibiliza a estudar esse conteúdo via um aplicativo?

Com relação à sequência didática, a aula foi ministrada com a mesma literatura utilizada pelo professor regente da turma, porém, com apresentações e auxílio do Geogebra mobile.

O GeoGebra mobile é oferecido pelo International GeoGebra Institute, lançado em 6 de dezembro de 2015 na Play Store. Segundo a página do aplicativo no Play Store, o GeoGebra mobile possui as seguintes funções:

- Escreva funções de plotagem, curvas polares e paramétricas;

- Experimente transformações com controles deslizantes;

- Obtenha pontos especiais de funções: raízes, min, max, interseções;

- Faça regressão com as linhas de melhor ajuste;

- Pesquise atividades de aprendizado gratuitas diretamente do nosso aplicativo;

- Salve e compartilhe seus resultados com amigos e professores.

Todos os alunos participantes tinham fácil acesso à internet para o download do aplicativo e a portabilidade de smartphones. Na Figura 1, apresenta-se a tela inicial do aplicativo GeoGebra mobile. 
Figura 1 - Tela inicial do GeoGebra mobile.

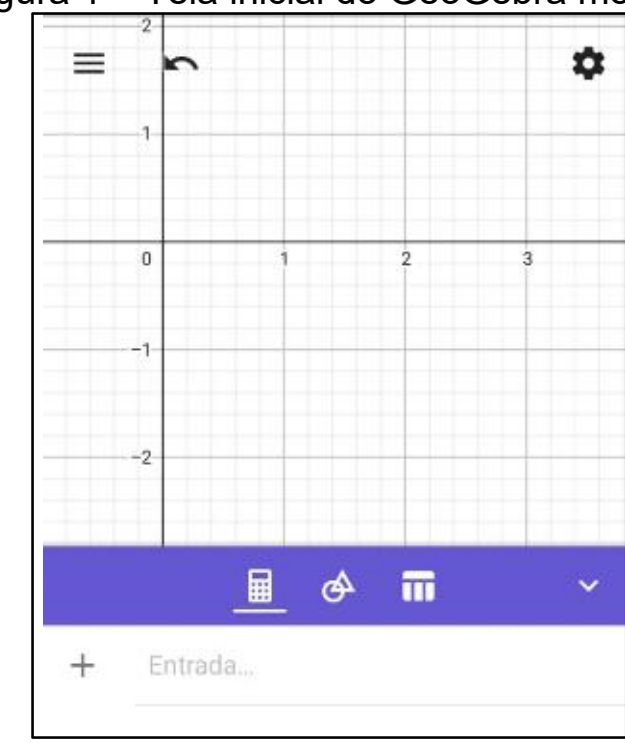

Fonte: Elaboração dos autores (2019).

No canto superior direito da tela inicial, ao abrir o aplicativo, encontra-se o local de acesso às configurações. Podemos ver, também na parte superior, uma "setinha" apontando para trás que representa a opção de desfazer. As três "barrinhas" na horizontal, localizadas no canto superior esquerdo, possuem algumas opções fornecidas pelo aplicativo. Logo abaixo, temos a representação do plano cartesiano para o esboço de funções digitadas e também uma barra azul onde aparecem, da esquerda para a direita: entrada, ferramentas e tabela de valores.

$\mathrm{Na}$ Figura 2 temos as opções que podem ser utilizadas pelo usuário do aplicativo. Essas opções aparecem ao clicar nas três barrinhas na horizontal descritas na Figura 1.

Figura 2 - Opções oferecidas pelo GeoGebra.

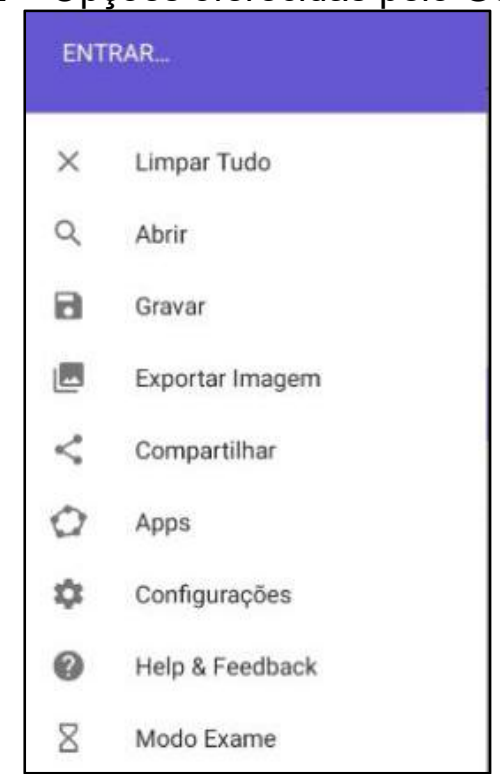

Fonte: Elaboração dos autores (2019).

Assim como na Figura 2, a Figura 3 detalha as configurações do GeoGebra. 
Figura 3 - a) Opção de configuração do GeoGebra em uma perspectiva geral; b) Opção de configuração para a janela de visualização; c) Opção de configuração relacionada à álgebra do aplicativo.

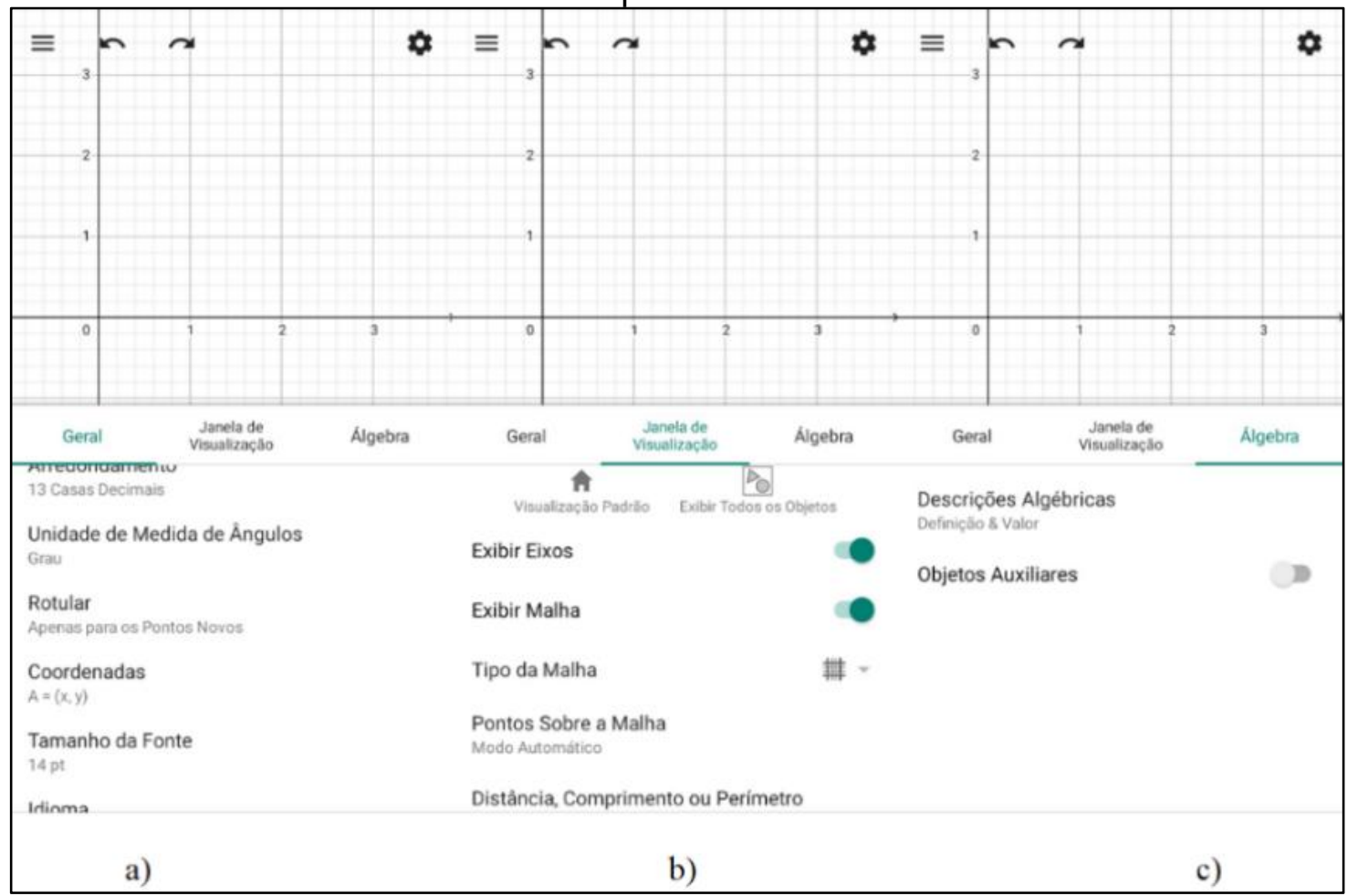

Fonte: Elaboração dos autores (2019).

Nessa Figura 3a), podemos ver que o aplicativo mostra muitas opções de configurações, relacionadas à configuração geral, sendo possível alterar a unidade de medida de ângulos, o rótulo, o tamanho da fonte, entre outros. Já na Figura 3b), é possível modificar o tipo de visualização do gráfico, com opções da exibição dos eixos, ou até o tipo de malha, entre outros, e também outras funções, voltadas à visualização no aplicativo. Por último, a Figura 3c) apresenta a opção de modificar as descrições algébricas e ativar os objetos auxiliares.

A Figura 4 mostra detalhes das ferramentas disponíveis, sendo a parte mais importante nesta pesquisa.

Figura 4 - a) Opções de ferramentas básicas; b) Opções de editar e de medições; c) Opções de transformar e de construções; d) Opções para as retas e círculos e ainda opções adicionais ao clicar em MAIS.

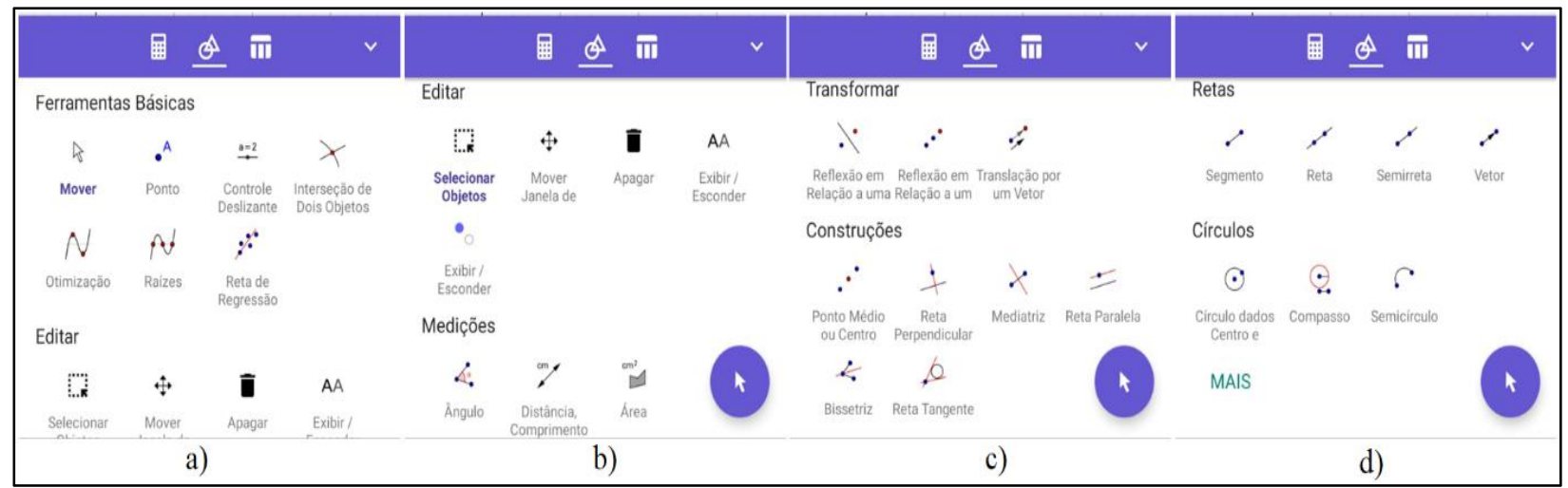

Fonte: Elaboração dos autores (2019). 
Na Figura 4, podemos ver à esquerda o desenho da calculadora, primeira opção na barra azul que foi mostrado na Figura 1. Em "Entrada" (Figura 1), é onde podemos digitar as funções que desejamos estudar. À direita, temos o ícone para a tabela que pode nos servir resultados dos pontos ao qual desejamos traçar, como mostrado na Figura 5.

Figura 5 - a) Função digitada na entrada e selecionados os três pontinhos verticais no canto superior direito, na mesma linha da função; b) Opção da barra de ferramentas azul, tabela de valores.

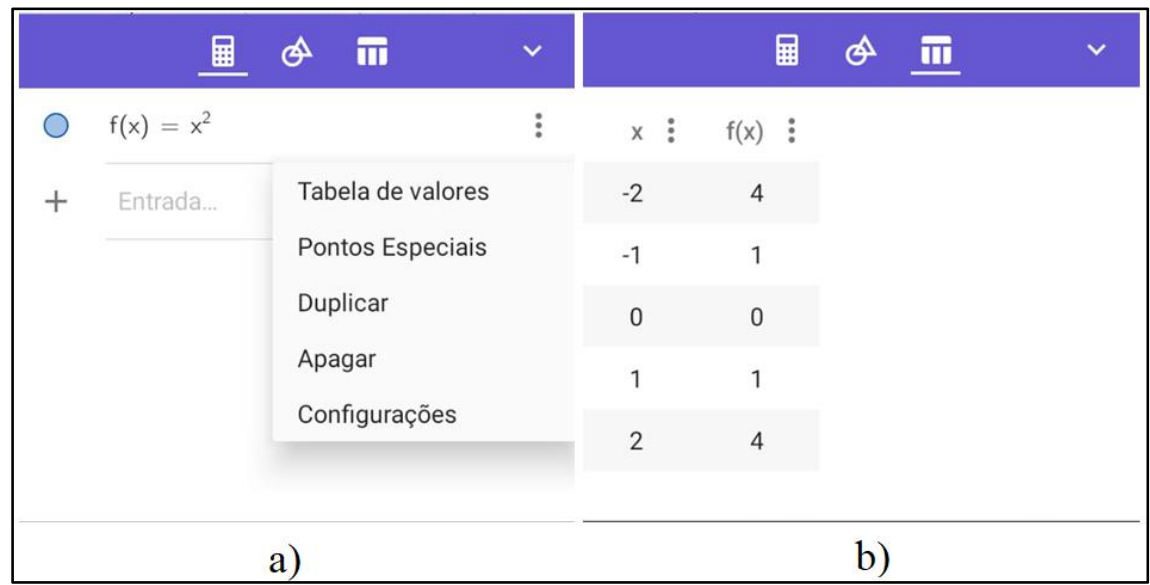

Fonte: Elaboração dos autores (2019).

Podemos perceber na Figura 5a) que há uma função como entrada ( $x^{2}$ ); temos muitas opções disponíveis como selecionar valores para a tabela de valores, selecionar pontos especiais, duplicar, apagar e configurar. Na Figura $5 b$ ), há a exibição de valores selecionados na tabela de valores. Esse aplicativo nos dá também a opção de adicionarmos mais de uma função, como as funções $f(x)$ e $g(x)$ da Figura 6.

Figura 6 - Duas funções na mesma janela gráfica.

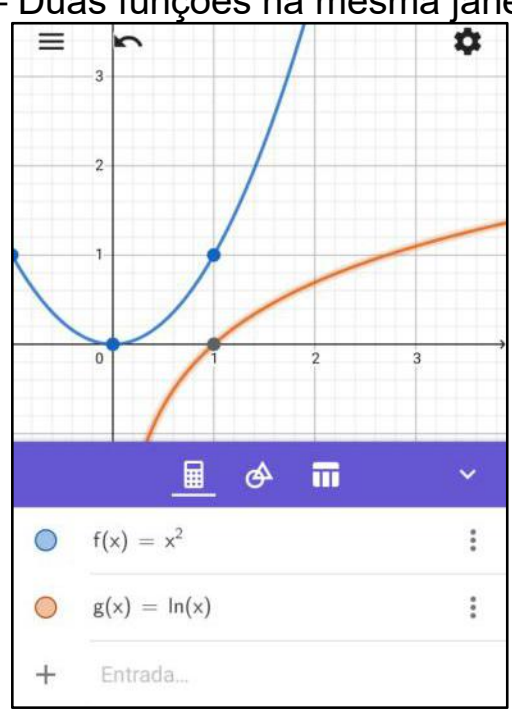

Fonte: Elaboração dos autores (2019).

Vale ressaltar ainda que podem ser adicionadas quantas funções o usuário desejar, e que também é possível definir a tabela de valores para cada função.

O pós-teste será detalhado na próxima seção. 


\section{Resultados e discussões}

Como dito na metodologia, inicialmente foi aplicado um pré-teste, que contou com a participação de 29 alunos; desses, 15 se disponibilizaram como voluntários para participarem de uma aula, ocorrida no mesmo horário da aula da disciplina a qual os alunos estavam matriculados, ministrada por outro professor (sendo esse também um professor da disciplina de Cálculo Diferencial e Integral), e essa aula sendo com o mesmo conteúdo abordado pelo professor da turma. Ao aplicar o pré-teste, nenhum dos alunos que se voluntariaram teve contato com o assunto anteriormente, todos eles estavam vendo o conteúdo pela primeira vez. $\mathrm{E}$ também, eles tinham grande expectativa de que seria um método diferente e mais eficiente.

Para o entendimento do funcionamento do aplicativo GeoGebra mobile, bastava ter um pouco de conhecimento sobre funções e noções básicas de como mexer em um smartphone. Assim, com todos os procedimentos aplicados, pudemos perceber uma grande interatividade e interesse dos alunos para aprender a manusear o GeoGebra, corroborando com Oliveira et al. (2012) que afirmam que, com tais recursos, se proporciona um ambiente interativo com informações em tempo real, dinamizando e enriquecendo o processo de ensino e aprendizagem.

$\mathrm{Na}$ aplicação da sequência didática, o conteúdo de funções inversas e logarítmicas foi apresentado iniciando com uma motivação dada pelo uso desses conceitos no mercado financeiro. Ao fornecer uma definição, essa foi explicada com a função descrita no GeoGebra mobile e cada estudante acompanhou com seu smartphone. Ao calcular a inversa de uma função, seus gráficos foram plotados e verificados, mostrando o que acontece ao cometer equívocos no cálculo. Ao trabalhar com logaritmos, suas propriedades também foram trabalhadas com o auxílio gráfico.

Durante a aplicação, notamos que o conteúdo era melhor compreendido quando se definia o assunto utilizando a análise algébrica e gráfica com exemplos e exercícios de fixação. Esses fatos estão em acordo com Lopes e Santos (2016) que sugerem que o ensino não seja praticado apenas na forma tradicional, reforçando que o ensino deve ser passado de forma que se torne mais interessante e menos repetitivo. Isso faz com que os resultados para alunos e professores sejam melhores utilizando alternativas diferentes, que é o caso desta intervenção que utilizou o GeoGebra na apresentação do conteúdo favorecendo uma adequada apropriação de conhecimentos.

Após a execução da sequência didática, foi aplicado o pós-teste, composto de duas questões sobre funções inversas e logarítmicas e perguntas de autoavaliação e satisfação quanto à metodologia utilizada. A Figura 7 mostra a autoavaliação dos alunos quanto ao seu aprendizado. O gráfico mostra que os alunos afirmaram ter boa aprendizagem sobre as funções inversas e logarítmicas, quando auxiliados pelo GeoGebra. Quanto à expectativa por outras atividades que utilizem a mesma metodologia, $80 \%$ dos discentes responderam que esperam ter outras aulas dessa natureza e $20 \%$ afirmaram que depende do conteúdo a ser trabalhado. 
Figura 7 - Autoavaliação dos estudantes quanto ao seu aprendizado individual.

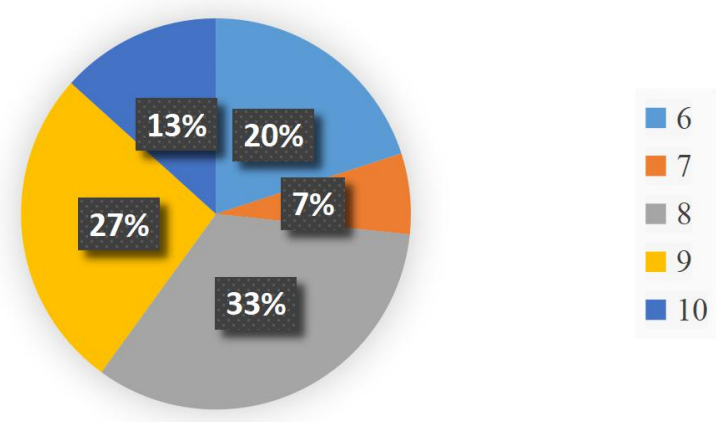

Fonte: Elaboração dos autores (2019).

A Figura 8 mostra, do ponto de vista dos estudantes, o quanto o GeoGebra com a metodologia aplicada contribuiu para o fortalecimento dos assuntos abordados.

Figura 8 - Percepção dos estudantes quanto à contribuição das atividades vivenciadas auxiliadas pelo GeoGebra.

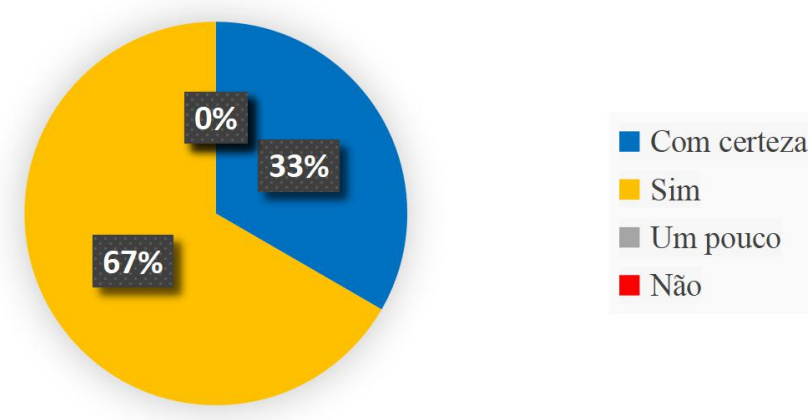

Fonte: Elaboração dos autores (2019).

No gráfico da Figura 8 observa-se que, ao menos em partes, o GeoGebra deu um fortalecimento maior para se entender os conteúdos estudados, em que se destaca que a maioria associa o êxito à metodologia experimentada por meio do GeoGebra. Esse êxito é obtido pois o uso do GeoGebra permite encorajar o processo de descoberta e autoavaliação, como afirmado por Lopes (2011) ao investigar o ensino de trigonometria.

A Figura 9 mostra a percepção dos alunos quanto à interatividade e facilidade de manuseio do aplicativo.

Figura 9 - Percepção dos estudantes quanto à interatividade e facilidade de manuseio do Geogebra, respectivamente.

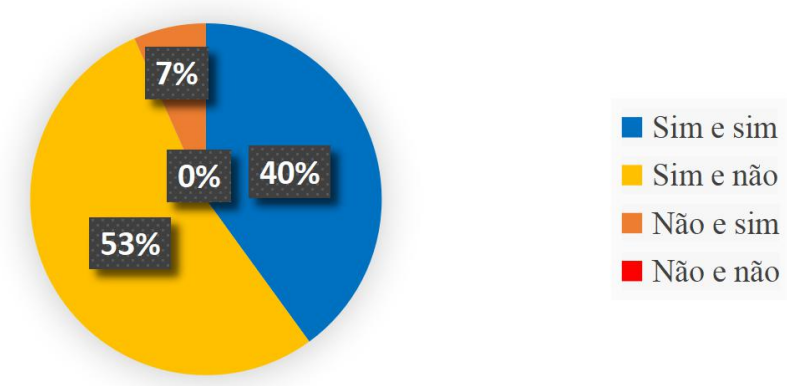

Fonte: Elaboração dos autores (2019). 
O gráfico da Figura 9 mostra que para os estudantes o aplicativo é bastante interativo e o manuseio é fácil para $40 \%$ e outros $53 \%$ acreditam que requer um pouco de prática. Todos os discentes afirmaram que o fato de o aplicativo ser em uma plataforma mobile ajudou para o contato e uso. Quanto à motivação, todos os discentes concordaram que o aplicativo os motiva a estudar os conteúdos de funções inversas e logarítmicas.

A seguir, são apresentados alguns comentários que foram coletados do pós-teste dos alunos:

Estudante A: - Auxilia no entendimento mais amplo dos gráficos na hora do estudo, nos permitindo uma visão melhor, dessa forma há um maior incentivo na hora de estudar. Seria interessante o uso em Geometria Analítica.

Estudante B: - Ajuda a tirar dúvidas na hora de confirmar o comportamento de uma função, por exemplo, quando preciso saber se o gráfico que fiz está com o comportamento certo. Serve até mesmo para funções bastante complexas. Tenho certeza que esse aplicativo me servirá para todo decorrer do curso.

Podemos perceber que os alunos gostaram bastante do uso do GeoGebra mobile para auxiliar nas disciplinas, tornando-as mais intuitivas e motivadoras. Esses comentários estão de acordo com Lopes (2011) que afirma que o GeoGebra nos possibilita pensar de uma forma matematicamente diferente do que se estivéssemos trabalhando com uma construção estática ou apenas falando dela, sem nenhum recurso visual, bem como com Lopes e Santos (2016) que, ao utilizar o GeoGebra no estudo da reta tangente, ressaltam que os discentes, motivados pelo uso do software, mostraram-se participativos e atuantes durante as atividades.

$\mathrm{Na}$ Figura 10, são apresentados dois exemplos de respostas às questões propostas no pós-teste.

Figura 10 - a) Resolução do teste do Estudante C; b) Resolução do teste do Estudante D.

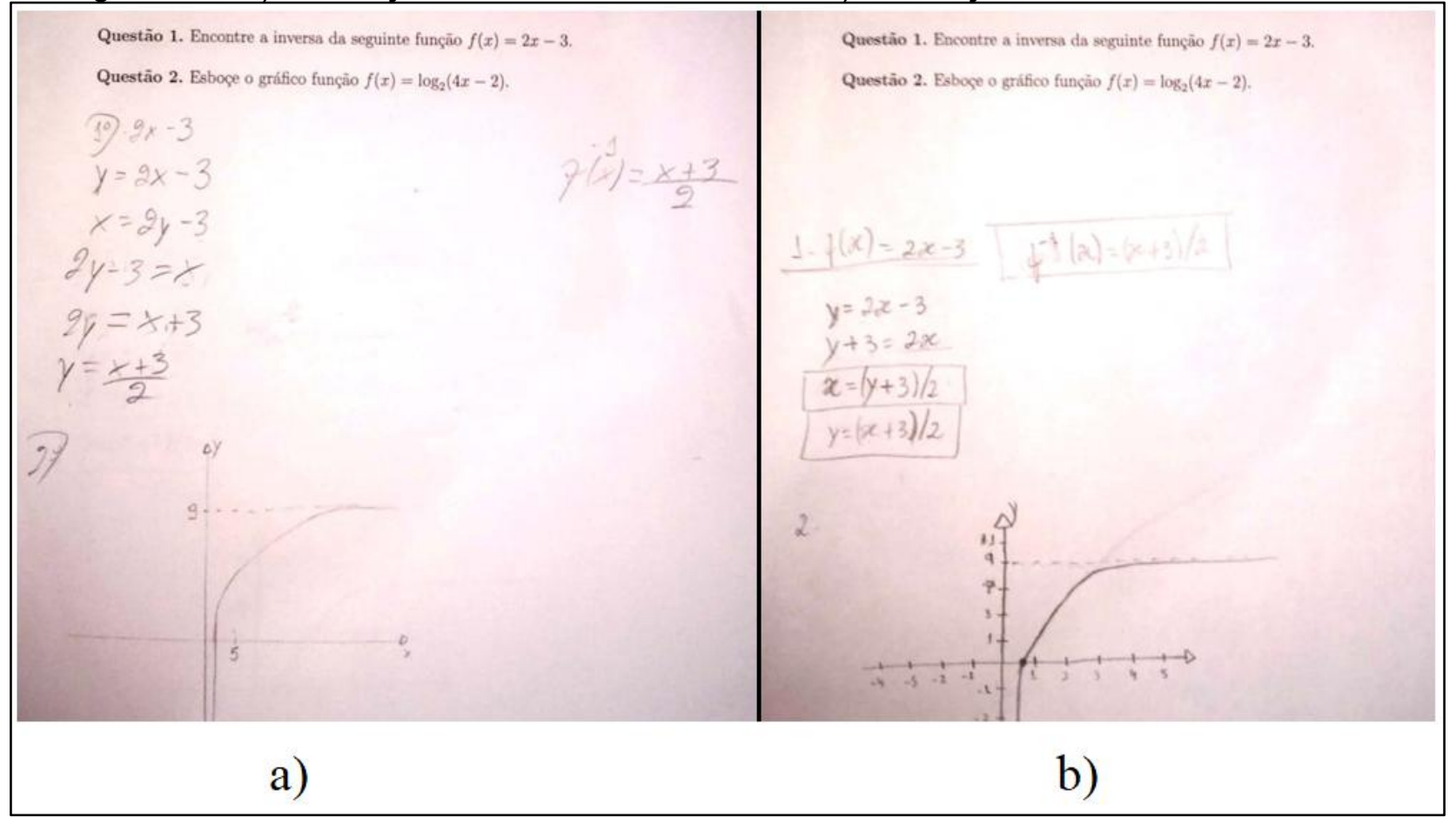

Fonte: Elaboração dos autores (2019). 
É possível observar, pela Figura 10, que os alunos conseguiram resolver com clareza e objetividade o teste proposto. Alguns alunos ficaram surpresos com a precisão dos resultados, pois ao inserir no Geogebra os gráficos dos alunos comparados ao do software foram fiéis um ao outro. E ainda, as soluções apresentadas mostram que o estudante compreende a noção de função inversa assimilando que esta tem domínio no conjunto imagem da função original e o esboço gráfico da função logarítmica foi realizado corretamente, fatos não verificados nos testes dos alunos que não participaram das aulas auxiliadas pelo GeoGebra.

A maioria dos alunos que participaram de toda metodologia aplicada conseguiram responder o pós-teste com facilidade.

\section{Considerações finais}

Nesta pesquisa, apresentamos uma sequência didática para o ensino de funções inversas e logarítmicas, na qual um objeto de aprendizagem é utilizado para auxiliar o processo, e para deixar a aula mais intuitiva e dinâmica possível. O estudo teve como alvo principal um grupo de estudantes calouros do Curso de Ciência e Tecnologia de uma instituição de Ensino Superior da região do Semiárido Potiguar no Rio Grande do Norte. Os estudantes que participaram da pesquisa a fizeram de forma voluntária e mostraram-se bastante motivados para o trabalho.

Vale ressaltar que o uso do GeoGebra vem cada vez mais ganhando espaço quando se trata de uma melhoria no ensino-aprendizagem. Nosso trabalho tem o intuito de mostrar que o uso desse aplicativo traz bons resultados e que o uso dele motiva os alunos a estudarem um pouco mais, por deixar alguns conteúdos mais intuitivos. Ao aplicar o teste avaliativo para os dois grupos, vimos que a maioria dos alunos participantes dessa nova experiência conseguiu responder as questões que estavam vinculadas ao aplicativo facilmente, embora alguns tenham demonstrado alguma dificuldade. Já os alunos que tiveram aula da forma tradicional, quase nenhum conseguiu resolver o teste.

É importante destacar também que a partir da pesquisa verificou-se que os estudantes gostariam que o mesmo procedimento fosse aplicado em outros momentos e até mesmo em outras disciplinas. Isso mostra o quão motivante foi a utilização do aplicativo GeoGebra mobile como um auxílio para a aprendizagem de funções inversas e logarítmicas.

Compreendemos que as alternativas devem ser buscadas e inseridas como prática docente para que os discentes possam experimentar, e que não se predomine apenas a apresentação e transmissão de conteúdo pelo professor. 


\section{Referências}

ARAÚJO, L. C. L. de; NÓBRIGA, J. C. C. Aprendendo matemática com o geogebra. São Paulo: Editora Exato, 2010.

FAVA, R. O Ensino na Sociedade Digital. SEMESP, São Paulo, 13 nov. 2012. Disponível em: http://www.semesp.org.br/noticias/o-ensino-na-sociedade-digital/. Acesso em: 23 set. 2019.

FRANCO, V. N. D.; PORTO, M. B. D. da S.; ALMEIDA, L. A. O desafio de inserção das tecnologias digitais na escola básica contemporânea. e-Mosaicos: Revista Multidisciplinar de Ensino, Pesquisa, Extensão e Cultura do Instituto de Aplicação Fernando Rodrigues da Silveira, Rio de Janeiro, v. 5, n. 10, p.14, dez. 2016. DOI: https://doi.org/10.12957/emosaicos.2016.26617.

FEITOSA, Murilo Carvalho; AQUINO, Adelmo Artur de; LAVOR, Otávio Paulino. Ensino de retas e planos com auxílio do software GeoGebra 3D mobile. Reamec: Rede Amazônica de Educação em Ciências e Matemática, Cuiabá, v. 8, n. 2, p. 374-391, 5 jun. 2020. DOI:

https://doi.org/10.26571/reamec.v8i2.10042.

LIMA, E. L.; CARVALHO, P. C. P.; WAGNER, E.; MORGADO, A. C. A Matemática do Ensino Médio. v. 1. 11. ed. Rio de Janeiro: SBM, 2016. Coleção Professor de Matemática.

LOPES, M. M. Contribuições do software geogebra no ensino e aprendizagem de trigonometria. In: CONFERÊNCIA INTERAMERICANA DE EDUCAÇÃO MATEMÁTICA, XIII, Recife, jun. 2011.

Anais..., Recife, 2011. Disponível em: https://xiii.ciaemredumate.org/index.php/xiii ciaem/xiii ciaem/paper/view/2577. Acesso em: 16 ago. 2020.

LOPES, T. B.; SANTOS, L. G. dos. O uso do GeoGebra como ferramenta auxiliar para estudo da reta tangente a um gráfico. RENOTE: Revista Novas Tecnologias na Educação, Porto Alegre, RS, v. 14, n. 2, dez. 2016. DOI: https://doi.org/10.22456/1679-1916.70637.

MATTAR, J. Aprendizagem em ambientes virtuais: teorias, conectivismo e MOOCs. TECCOGS: Revista Digital de Tecnologias Cognitivas, São Paulo, n. 7, p. 21-40, jan./jun. 2013. Disponível em: http://www4.pucsp.br/pos/tidd/teccogs/artigos/2013/edicao 7/2aprendizagem em ambientes virtuais-joao mattar.pdf. Acesso em: 23 jul. 2020.

MEIER, M.; GRAVIRA, M. A. Modelagem no GeoGebra e o desenvolvimento do pensamento geométrico no Ensino Fundamental. Revista do Instituto GeoGebra Internacional de São Paulo, v. 1, n. 1, p. CCL-CCLXIV, maio 2012. Disponível em:

http://ken.pucsp.br/IGISP/article/view/9583/7124. Acesso em: 20 set. 2019.

MOLINARI, J. R. A.; SANTOS, L. A. dos; RETSLAFF, F. M. de S. Um relato de experiência no ensino de funções quadráticas com a utilização do software GeoGebra. REMAT: Revista Eletrônica da Matemática, Bento Gonçalves, v. 5, n. 2, p. 15-28, 1 jul. 2019. DOI: https://doi.org/10.35819/remat2019v5i2id3287.

NASCIMENTO, E. G. A. do. Avaliação do uso do software GeoGebra no ensino de geometria: reflexão da prática na escola. In: CONFERENCIA LATINOAMERICANA DE GEOGEBRA, nov. 2012, Montevideo, Uruguay. Actas... Montevideo, Uruguay, 2012. Disponível em: http://www.geogebra.org.uy/2012/actas/procesadas1370724062/67.pdf. Acesso em: 9 out. 2019. 
OLIVEIRA, J. B. de; SANTANA, A. M.; REALI, G. A.; OLIVEIRA, M. C. D. de; SILVA, D. L.; QUEIROZ, F. N. de. O uso de tablets e o GeoGebra como ferramentas auxiliadoras no ensino da Matemática. In: CONFERENCIA LATINOAMERICANA DE GEOGEBRA, nov. 2012, Montevideo, Uruguay. Actas... Montevideo, Uruguay, 2012. Disponível em:

http://www.geogebra.org.uy/2012/actas/procesadas1369046973/66.pdf. Acesso em: 26 set. 2019.

PETLA, R. J. GeoGebra: possibilidades para o ensino de Matemática. 2008. 45f. Trabalho de Conclusão de Curso (Graduação em Matemática) - Departamento de Matemática, Universidade Federal do Paraná, União da Vitória, 2008.

REZENDE, W. M.; PESCO, D. U.; BORTOLOSSI, H. J. Explorando aspectos dinâmicos no ensino de funções reais com recursos do GeoGebra. Revista do Instituto GeoGebra Internacional de São Paulo, v. 1, n. 1, p. 74-89, mar. 2012. Disponível em:

http://ken.pucsp.br/IGISP/article/view/8370/6580. Acesso em: 9 out. 2019. 